\title{
CONCEPCIONES PREVIAS EN EL APRENDIZAJE SIGNIFICATIVO DEL ELECTROMAGNETISMO
}

\author{
COLOMBO DE CUDMANI, L. y FONTDEVILA, P.A. \\ Instituto de Física, Universidad Nacional de Tucumán.
}

\section{SUMMARY}

Difficulties that are present in the basic conceptualizations of electromagnetism are analized.

The logic way, followed in physics instruction, which is not necessarily the most adequate to initiate the study of the subject is emphasized. The psychological importance of the previous concepts is also considered.

As a consequence the investigation is faced through inquiries, observations, and data accumulation along the teaching experience on the most frequent preconceptions.

The statistical work has been carried out with students of secondary level and university college.

As a conclusion subsumers are proposed, which appear as the most adequate to the study of electromagnetism courses content structuration.

\section{INTRODUCCIÓN}

La experiencia docente muestra que, en general, el aprendizaje del electromagnetismo tanto en el ciclo de enseñanza media como en los niveles básicos universitarios ofrece serias dificultades a los estudiantes. Estas dificultades se ponen de manifiesto fundamentalmente en los aspectos conceptuales de la disciplina.

Son diversas las fuentes de esas dificultades pero en base a nuestras observaciones formulamos la hipótesis de que parte del problema radica en la tradicional estructuración de los contenidos.

Sobre esta base y trabajando en forma empírica se diseñó un nuevo esquema secuencial, que fue propuesto a un grupo de profesores secundarios durante un curso de perfeccionamiento (1978).

Un seguimiento posterior mostró que los resultados habían mejorado sensiblemente. Sin embargo, estas conclusiones tenían fuertes características intuitivas y no sistemáticas y el esquema mismo carecía de fundamentos teóricos que permitieran perfeccionarlo y dinamizarlo.

Se trata ahora de llevar la propuesta empírica a un plano racional, con respaldo teórico en las ciencias de aprendizaje, lo que permitiría hacer un análisis sistemático y científico de esta problemática.

\section{EL ENFOQUE TRADICIONAL Y SUS LIMITACIONES}

Habitualmente la enseñanza del electromagnetismo se ha encarado, en textos y problemas, a partir de la Electrostática, tomando como estructuras conceptuales de partida los conceptos de carga, campo y potencial. El hilo estructural va de lo simple a lo complejo con un criterio logico comprensible y racional para la mente del científico entrenado.

Cabe preguntarse si este camino, en cierta manera obvio dentro de los cánones de la construcción de una disciplina científica, es también el más adecuado para un eficiente aprendizaje (Halbwachs 1985).

¿Son los conceptos simples los de más facil asimilación? ¿Se adecuan al estado evolutivo del desarrollo del pensamiento formal del estudiante? (Piaget 1979). ¿Están suficientemente próximos a la estructura cognoscitiva del alumno como para permitir un aprendizaje significativo? ¿Tienen suficientes correlatos en las experiencias vitales como para motivarlos? Si se tiene en cuenta que estos conceptos «simples» son en realidad resultado de complejas abstracciones no parece tan claro que sean buenos puntos de partida para estructurar un nuevo campo de aprendizaje.

Si bien es cierto que históricamente, en las primeras etapas del estudio de los fenómenos electromagnéticos 
(EM), existía correspondencia entre los aspectos fenomenológicos y la conceptualización sistemática, esto ha perdido vigencia para el joven de hoy, inmerso en una tecnología donde los conceptos de corriente, tensión, energía integran su vocabulario desde la infancia.

Por otro lado, los clásicos problemas de aplicación en el tema, donde se dan configuraciones geométricas estáticas de carga y se pide el campo o el potencial en algún punto, o las caprichosas configuraciones de cargas totalmente alejadas de su experiencia contribuyen a generar en los alumnos actitudes de rechazo difíciles de superar.

No parecen, por cierto, las situaciones problemáticas más adecuadas para plantear en las primeras etapas de aprendizaje del EM.

Situaciones análogas aparecen cuando se parte de la Estática o la Cinemática en la enseñanza de la Mecánica, lo cual ha llevado a los docentes a nuevas organizaciones de contenidos (Feynman 1963, Cudmani 1978 y Kraushear 1966).

Comenzaremos pues por establecer criterios que permi tan seleccionar el o los núcleos conceptuales más eficientes que sirvan de base para estructurar los contenidos de la disciplina.

\section{IMPORTANCIA DE INVESTIGAR LAS ESTRUCTURAS CONCEPTUALES PREVIAS}

Dentro del modelo de aprendizaje operativo con el cual se ha venido trabajando desde 1976 (Cudmani y Lewin 1987) se integra muy bien el modelo de Ausubel (1973) cuando se trata de organizar la enseñanza de una disci. plina científica en el aula. Como la estructuración de los contenidos es un aspecto de dicha organización, tomaremos su modelo como base, pero sin dejar de tener en cuenta los aportes positivos que pueden extractarse de otros modelos, sean o no operativistas.

Como bien señala Ausubel (1973), «además de los cuerpos de conocimiento organizados (disciplinas científicas) existen estructuras psicológicas de conocimiento... representadas por la organización de las ideas y la información internalizada en la mente de los estudiantes".

Para que los nuevos contenidos puedan ser significativamente aprendidos y retenidos es necesario que se hallen disponibles en la estructura cognoscitiva conocimientos o conceptos más inclusivos y relevantes que proporcionen un «anclaje conceptual» a las nuevas ideas. Son los «inclusores» (o subsunsores) que mediante el proceso de acreción determinan la adquisición de nuevos significados y su retención. En nuestro trabajo usamos la traducción subsunsor (en lugar de inclusor). ${ }^{*} \mathrm{Y}$ distinguiremos el significado lógico o potencial característico del material de aprendizaje, del significado psicologico.
* Aun cuando se trate de un neologismo, pues refleja mejor el proceso de acreción que describe Ausubel y en el cual hay una reelaboración del concepto primitivo, que va más allá de una mera «inclusión».

Este significado lógico o potencial «está integrado por relaciones posibles y no arbitrarias que puedan relacionarse... con una estructura cognoscitiva humana hipotética que posea los fundamentos racionales y la madurez cognoscitiva necesarios" (Ausubel 1973).

La inclusión de las proposiciones lógicamente significativas en una estructura cognoscitiva particular es la que permite transformar el significado lógico en psicológico.

Tanto el proceso lógico como el psicológico se ajustan a los principios de la "clasificación», en tanto pueden considerarse procesos de inclusión en aquellos conceptos relevantes ya existentes. Podríamos pues, extractar como características importantes de los subsunsores:

I. preexistencia en la estructura cognoscitiva

alto grado de generalidad

alto grado de inclusividad

poder explicativo

Con estas condiciones, estos subsunsores serán capaces de relacionar e integrar el más amplio dominio posible de la materia.

Pero desde el punto de vista psicológico, este proceso de inclusión que inicialmente facilita tanto el aprendizaje como la retención, Ileva a un segundo estadio, la asimilación obliteradora (Ausubel 1973) en donde un nuevo concepto más inclusivo reemplaza al inclusor inicial.

La resistencia a este decrecimiento es función de propiedades cognoscitivas de los subsunsores con respecto al nuevo material, tales como:

\section{II. asequibilidad \\ estabilidad \\ claridad \\ capacidad discriminativa}

Parecería pues, que las características destacadas en I y II serían desideratas que permitirían seleccionar, dentro de los subsunsores disponibles, los más aptos para organizar el aprendizaje.

La importancia de una investigación previa de cuáles son esos subsunsores parece ampliamente justificada.

Aunque expresada en otros términos o analizadas desde distintos puntos de vista, otros modelos de aprendizaje rescatan esta relevancia.

Cuando Gagné analiza las condiciones específicas para 
que se dé el aprendizaje menciona entre las «condiciones internas", "capacidades preexistentes en el individuo que deben ser recuperadas cada vez que se realiza un nuevo aprendizaje» (Moreira 1983).

Brunner, en su importante consideración sobre que «los fundamentos de cualquier conocimiento pueden ser enseñados a cualquiera», Ia condiciona a que para ello estos conocimientos deben organizarse «de algún modo reconocible por el que aprende», "que nunca parte de cero... y consiste en una diferenciación a partir de esquemas anteriores» (Ausubel 1973).

En el modelo de Posner (Posner et al. 1982), el aprendizaje en ciencias es un proceso de cambio conceptual, donde una estructura conceptual es cambiađa por otra incompatible con la anterior. Pero para que el aprendizaje tenga permanencia la nueva estructura debe tener por lo menos el mismo grado de coherencia interna y "ser elaborada de acuerdo a su experiencia pasada». El núcleo del modelo consiste en interpretar el proceso EA como una interacción entre las conceptuaciones que el alumno posee y la nueva información que incorpora. Parece clara, pues, la coincidencia en todas estas formulaciones, respecto a la importancia de las conceptualizaciones o de la estructura cognoscitiva preexistente.

Hasta aquí hemos analizado la importancia de detectar y seleccionar con criterios válidos los subsunsores que servirán de base a la estructuración del contenido en función de su capacidad positiva de servir de anclaje a las nuevas informaciones.

Sin embargo esta investigación ofrece otro valor para la tarea docente: la de poner en evidencia los preconceptos, las concepciones ingenuas e infantiles del pensamiento pre-lógico (Halbwachs 1985), fuente de las más habituales confusiones y falsas concepciones. Numerosas investigaciones en los últimos años han mostrado la resistencia de estas concepciones a la instrucción tradicional, fundamentalmente en el campo de la mecánica (Piaget 1973, Viennot 1979, Clement 1982, Solís Vila 1984).

Posner (op. cit.) analiza algunas sólidas razones para esa persistencia: primordialmente porque estos esquemas intuitivos constituyen estructuras comprensibles y cohe. rentes que sólo serán sustituidas por otras estructuras igualmente comprensibles y coherentes que den res. puesta a problemas que la estructura anterior no resolvía, tal es el caso del bien estudiado problema de la persistencia del preconcepto que establece la proporcionalidad entre la fuerza y la velocidad en la mecánica.

Es interesante, pues, investigar estas concepciones y su grado de internalización en el área del Electromagnetismo.

De las observaciones empíricas podemos anticipar algu* nas de estas preconcepciones, muy difíciles de erradicar. Valga como ejemplo el de la interpretación de la co. rriente eléctrica como el movimiento de un fluido que se desplaza por el conductor. Esta analogía que se usa incluso para explicar algunos fenómenos eléctricos lleva a explicaciones contradictorias cuando se trata de explicar, por ejemplo, que no haya acumulación de materia en un sólido que conduce corriente y sí la haya en un electrolito. O la «simultaneidad» entre el instante en que se enciende un foco luminoso y el instante en que se conecta la fuente, que puede estar muy alejada. Se llega así a aceptar que el «fluido» viaja a la velocidad de la luz.

Otros errores de este tipo derivan de la utilización en la vida cotidiana de un mismo término para designar conceptos que en el campo de la disciplina tiene un significado diferente. Como el conocido y estudiado caso del concepto de trabajo en la mecánica, se presentan en el electromagnetismo confusiones análogas entre tensión y energía de una fuente eléctrica, o con el concepto de capacidad y energía que puede suministrar un acumulador.

A fin de disponer de información más objetiva sobre las concepciones previas de los estudiantes sobre electromagnetismo, hemos realizado encuestas a distintos grupos de alumnos a las que nos referiremos en el siguiente parágrafo.

\section{UNA INDAGACIÓN DE CAMPO}

Buscando información objetiva sobre los conocimientos con que acceden los alumnos a la instrucción superior, se preparó una experiencia piloto en la forma de una consulta abierta cuya pregunta principal era: ¿Qué sabe Ud. sobre la electricidad? (ver Apéndice I). Respondieron 105 estudiantes de la Facultad de Ciencias Exactas y 103 de la Facultad de Bioquímica, Química y Farmacia, todos ingresantes en 1987 a la Universidad Nacional de Tucumán.

Por la naturaleza abierta de la consulta, el espectro de respuestas resultó muy amplio, y llamativa la similitud entre ambos grupos. Las dificultades de clasificación y aún de análisis de respuestas tan variadas, sin embargo, no impidieron distinguir las caracteráasticas idiosincráticas, en lo referido a tiempo y lugar, que condicionan las experiencias de la particular población estudiantil con que se trabajó. Y agrupadas por ítems, las respuestas que reflejaban una misma idea o preconcepto, permitieron preparar las 5 preguntas de opción que integran la encuesta propiamente dicha (Apéndice II). Esta última es cerrada a fin de manejar mejor los resultados, pero supone una pérdida de espontaneidad en las respuestas, pues el alumno tiene que optar por definiciones que quizá no le son propias.

Es interesante destacar que, si bien el cuestionario tiene la forma de ia opción múltiple (multiple choice) en cuanto ofrece opciones fijas para la respuesta, no se usa como un instrumento de evaluación, sino como una forma de indagar las ideas que el encuestado ha internalizado sobre el tema. De ahí que no haya necesariamente una respuesta correcta y que algunas preguntas no tengan una clara significación en el contex to de la ciencia.

Por eso la primera encuesta ha sido fundamental para proporcionar el material que permitiera elaborar la se- 
gunda, pues ésta respeta las ideas espontáneas que aparecieron con mayor frecuencia en la primera encuesta.

De todos modos, siempre se abre la posibilidad de una respuesta diferente a las propuestas, pues en la consigna inicial se dice «...en el caso de que ninguna de las opciones corresponde a lo que Ud. piensa, indíquela con otros.»

Para esta segunda encuesta fueron consuitados 112 estudiantes del Curso de Ingreso de 1988 de la Facultad de Ciencias Exactas y 136 de la Facultad de Bioquímica, Química y Farmacia de la UNT (Grupo 1), así como 83 alumnos de $4^{\circ}$ y $5^{\circ}$ año del Colegio Nacional Bartolomé Mitre (Grupo 2). Los resultados, resumen de los datos más significativos, se muestran en los cuadros que se reproducen al final del articulo.

El gráfico que sintetiza los resuliados de Ia pregunta ${ }^{2}$ 1 muestra la fuerte incidencia y permanencia del concepto energía. No se trata seguramente del concepto físico «energía» sino de un preconcepto con mucho arraigo en la experiencia previa, tanto en lo observacional como en el uso generalizado de la palabra (Viennot 1985).

La encuesta «abierta» (Apéndice I) mostraba que la concepción energía responde a las características que Piaget (1973, p. 187) asigna al pensamiento intuitivo: «la percepción de las relaciones es en líneas generales exacta, pero la construcción intelectual es incompleta... son configuraciones centradas sobre una relación y las relaciones que construye no son susceptibles de componerse entre sí» como también se verifica al avanzar este análisis.

Es por eso que en este trabajo hablaremos del preconcepto de energía o turbina o intensidad de corriente, etc. por entender que la idea con que manejan los alumnos estos términos poseen las características de las preconceptuaciones o ideas alternativas. Poco tienen que ver sus concepciones con los conceptos homónimos de la ciencia.

Consideramos que el preconcepto energía es un subsunsor válido para elaborar las conceptuaciones del electromagnetismo. Por sus características de generalidad e inclusividad, proporciona un útil anclaje para el inicio del aprendizaje sistemático y lógico del tema. Para que no se transforme en fuente de futuras confusiones, debe ser correctamente reelaborado.

Por su parte, Ia concepción de la electricidad como flujo de sustancia, que es la otra respuesta a la pregunta $\mathrm{n}^{\circ} 1$ con alguna significación (aunque mucho menos frecuente) es un preconcepto cuyas raíces podrían estar en las concepciones mecanicistas de la realidad, convalidadas por las analogías usuales con el movimiento de los líquidos.

La comparación de las respuestas dadas por los grupos 1 y 2 parecen demostrar sorprendentemente que este pre* concepto se arraiga con Ia instruccion. Por ello nos parece muy importante destacar que si ese tipo de analo- gías, demasiado usual en la práctica docente, no es correctamente elaborada constituye un serio obstáculo para las conceptualizaciones sobre el tema.

Veamos ahora las respuestas obtenidas para la pregunta $n^{\circ} 2$ de nuestra encuesta: los alumnos se inclinan nuevamente por opciones intuitivas o preconceptuales, percibiéndose incluso un ligero avance de lo preconceptual sobre lo intuitivo. Si bien el $48 \%$ del grupo I opta por el preconcepto turbina, frente al $30 \%$ del grupo 2, la presencia de ideas infantiles como sol o agua es muy notoria y muestran gran persistencia aún frente a la instrucción. Se podría hablar de un avance secuencial: agua o sol - dique - turbina. El paso de la escuela secundaria a la Universidad muestra sólo un ligero progreso. Y es interesante destacar que el alumno usa el término turbina en lugar de generador. Una parte, la más conocida y familiar ha reemplazado al todo, el concepto más complejo de generador.

En las respuestas a la pregunta $n^{8} 3$ se revela con claridad la importancia de las experiencias vitales (-iQué caro está el «kilovatio»!) hasta dejar de lado los efectos de la instrucción.

Es interesante destacar primero, que si bien la encuesta ofrecía como opción el kwh, nadie vinculó esta unidad con Ia energía y sí, en cambio, un $32 \%$ elige el kilowatt en su lugar. Vemos aquí un claro indicio de la persistencia de la concepción vulgar que mide el «gasto» de electricidad en kilovatios y no en la unidad de consumo que es el kwh.

Ahora bien, tal como se estructuran los cursos de Física en el nivel secundario, se toma el Coulomb como unidad fundamental más importante, y de la cual se derivan todas las restantes. Sin embargo, en el grupo I tiene baja frecuencia y en el grupo 2 esa unidad no aparece ¿Por qué es aquí tan resistida la instrucción? ¿Choca demasiado con la experiencia vital? El lugar del coulombio lo ocupa principalmente el voltio y el citado kilovatio, unidades familiares. Por eso, y porque representan uni* dades más fáciimente relacionables con la energía aparece reforzado este concepto como subsunsor más relevante que el de carga eléctrica.

Los gráficos representan la distribución porcentual de las respuestas a la pregunta $n^{2} 4$.

Lo más destacado aquí es que se pone de manifiesto la inestabilidad, incoherencia y contradicción en que incurre el razonamiento intuitivo o preconceptual. Porcentajes importantes de alumnos opinan que el voltio es la unidad de carga eléctrica, así como muchos dicen que en realidad es la unidad de la intensidad de corriente. Es que en este pensamiento prelógico «relación inmediata entre un esquema de acción interiorizada y la percepción de los objetos, la intuición no desemboca sino en configuraciones centradas sobre esa relación" y cuando aparece otro «centro» las relaciones no son susceptibles de com. ponerse entre sí. «El símbolo conceptual de la configuración intuitiva se halla siempre centrada en un estado particular del objeto y en un punto de vista particular del sujeto» (Piaget 1973 , p. 192). La descentración de am- 
bos es necesaria para que el pensamiento intuitivo pueda convertirse en operativo.

En nuestro caso, los centros energía, intensidad de corriente o carga son inestables y su relación entre sí o con otros provoca una contradicción muy acentuada y generalizada. Es notoria la gran dispersión de las repuestas y la baja proporción de las que podríamos llamar coherentes. Rescatamos no obstante el preconcepto Intensidad de Corriente Eléctrica que aparece compitiendo casi en paridad con el de energía en ambos grupos. Por tratarse de una conceptualización conectada específícamente con el campo de los fenómenos eléctricos se puede considerar otro subsunsor posible.

En el gráfico que presenta las respuestas a la pregunta ${ }^{2}$ 5 se confirman las observaciones hechas al estudiar las respuestas a la pregunta $n^{\circ} 2$. Una mayoría se inclina por los efectos calóricos y luminosos reflejando resultados de su experiencia cotidiana.

Asimismo, presentamos los resultados obtenidos en la pregunta $n^{\circ} 6$. Puede observarse otra vez la baja incidencia que tiene la instrucción en conceptos espećf́ficos del campo de la electrostática. Y por otro lado resalta el impacto de la tecnología que rodea a los jóvenes de hoy. Ésta provee de un vocabulario que prevalece por encima del libro de texto de la enseñanza sistemática. Valga como ejemplo la alta frecuencia con que eligen el «tester».

El carácter egocéntrico del pensamiento preconceptual e intuitivo se pone bien de manifiesto en los resultados que hemos comentado. Las experiencias vitales, el lenguaje ordinario, tos medios de comunicación de masas han jugado un papel primordial en la elaboración de las respuestas a nuestra encuesta, mientras la instrucción parece jugar un papel secundario.

Digamos por último que en la encuesta abierta preparatoria aparecen, aunque con baja incidencia porcentual, sorprendentes respuestas que muestran niveles infantiles de conceptuación, tales como considerara a la electricidad como «lo que ocurre cuando chocan dos nubes y producen un rayo» o cuando se cita como único ejemplo de los efectos de la electricidad al «cortocircuito». Si consideramos que se trata de estudiantes que ingresan a la Universidad, no puede menos que llamarnos la atención sobre ei nivel de desarrollo de operaciones formales que estas respuestas sugieren.

\section{CONCLUSIONES}

La encuesta confirma, en términos generales, Ios supuestos empíricos surgidos de la práctica docente que se mencionan al comienzo del trabajo, en el sentido de que las experiencias y conceptuaciones de la electrostática no parecen suministrar buenos subsunsores para el electromagnetismo en los cursos secundarios y básicos universitarios.

En efecto, del análisis de las encuestas se desprende que, pese a que la instrucción formal recibida parte del concepto de carga eléctrica y del coulombio como unidad fundamental más importante, en las respuestas de los estudiantes son muy pocos los que relacionan estas nociones con los fenómenos eléctricos en comparación con los que los vinculan con la energía y la corriente. Es muy posible que sus experiencias vitales en este campo estén, como ya se dijo, mucho más ligadas a las nociones de energía ("gasto») y corriente que son los que se vinculan más directamente con los «efectos»y las «aplicaciones», que a las nociones más abstractas de carga, fuerzas entre cargas, campo, etc.

De ahí que nuestra propuesta consista en tomar como concepto estructurador de las conceptuaciones del Electromagnetismo el concepto de Energía, y como subsunsor fundamental, el concepto de corriente eléctriça.

No cabe duda de que las nociones que los estudiantes tienen sobre estos dos conceptos son preconceptuales y por lo tanto, poco precisas, contradictorias y confusas. Es necesario elaborarlas cuidadosamente para llevarlas al nivel lógico pero sin despojarlas de los caracteres psicológicos y experienciales que le dan permanencia e internalización.

En un próximo trabajo se propone un esquema completo de estructuración de contenidos en cursos de Electromagnetismo, sobre la base de este estudio introductorio. En él se construyen redes conceptuales y diagramas secuenciales cuyo nucleo estructural es el concepto de energía, en primer término, y el de corriente eléctrica en segundo tugar.

\section{AGRADECIMIENTOS}

Los autores quieren dejar constancia de su reconocimiento al Dr. Antonio Moreira por haber leído los originales antes de su publicación. Asimismo por las sugerencias que tuviera la amabilidad de prestamos para mejorar la presentación de gráficos y conceptos.

Deseamos expresar nuestro agradecimiento a los señores profesores: Teresa M. De Lomáscolo, Patricia A. de Cozzitorti y Víctor $\mathrm{Kat} z$, por la colaboración prestada con las encuestas en el ciclo de enseñanza media y en la Facultad de Bioquímica, Química y Farmacia. 


\section{CONOCIMIENTOS PREVIOS}

¿Qué sabe Ud. sobre la «electricidad»?

¿Qué efectos conoce Ud. que produce la «electricidad»? APÉNDICE I

¿Qué fuentes de «electricidad» conoce?

¿Con qué se mide la «electricidad»? ¿En qué unidades?

¿Qué es para Ud. la «electricidad»? una sustancia

ana energía

una fuerza

¿De qué sustancias se hacen los cables? ¿Sabe por qué?

¿De qué sustancias se hacen las llaves de la luz, las fichas de planchas, los terminales para conectar? ¿Sabe por qué?

\section{APÉNDICE II \\ ENCUESTA}

En cada una de las siguientes proposiciones se le ofrecen diversas alternativas para la respuesta. Marque con un recuadro la opción que, a su juicio, mejor se ajusta. Sólo puede elegir una opción; en el caso de que ninguna de las opciones corresponda a lo que Udd. piensa, indfquela en otros.

1.- La mejor descripción de la electricidad es considerarla: $\left(^{*}\right)$
a) un campo
b) una sustancia que se mueve
c) una fuerza
d) energía

2.- La principal fuente de electricidad en la vida diaria es:
a) sol
b) agua
c) diques
d) turbinas
e) pilas

3.- La electricidad se mide en:
a) kilovatios
b) voltios
c) amperios
d) coulombios
e) kilovatio hora
f) omhios
g) joute

4.- La unidad que Ud. eligió en la pregunta $n^{2} 3$ corresponde a la magnitud:
a) intensidad de corriente
b) carga eléctrica
c) energía eléctrica
d) diferencia de potencial
e) potencia eléctrica

S.- ¿En cuál de los siguientes casos diría Ud. que circula electricidad por un conductor?
a) la aguja de una brujula muy próxima sufre una desviación
b) se calienta
c) emite luz
d) se producen cambios químicos

6. - El instrumento para medir electricidad se liama:
a) electroscopio
b) voltímetro
c) amperímetro
d) vatímetro
e) voltámetro

(*) Las opciones «usina» o «generador» no se incluyeron en esta encuesta en razón de que no aparecieron, en forma espontánea, en las respuestas de la primera encuesta (Apéndice 1). 


\section{RESPUESTAS A LAS PREGUNTAS}

Respuesta a la pregunta $n^{\circ} 1$

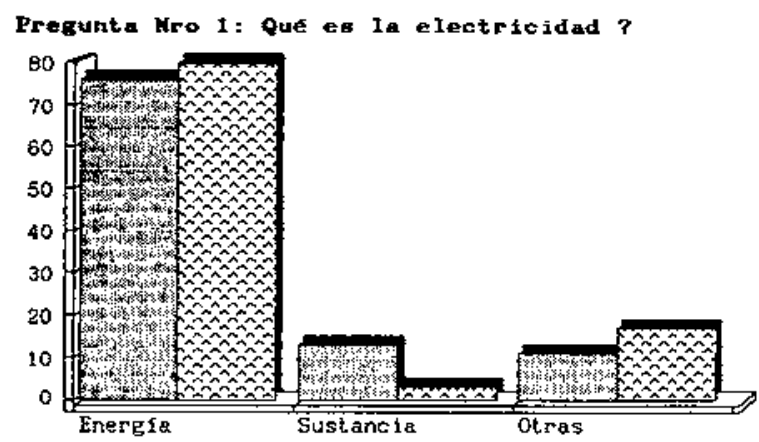

泼 610

Respuesta a la pregunta n 2

Pregunta Mro 2: La fuente de Ia electricidad es...

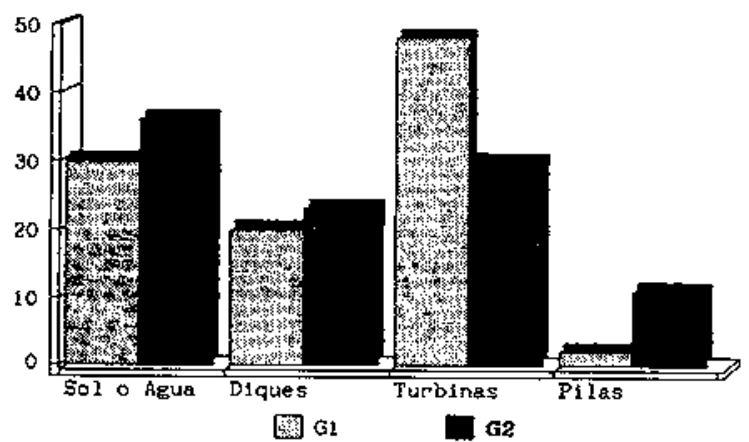

Respuesta a la pregunta n⿳0 3

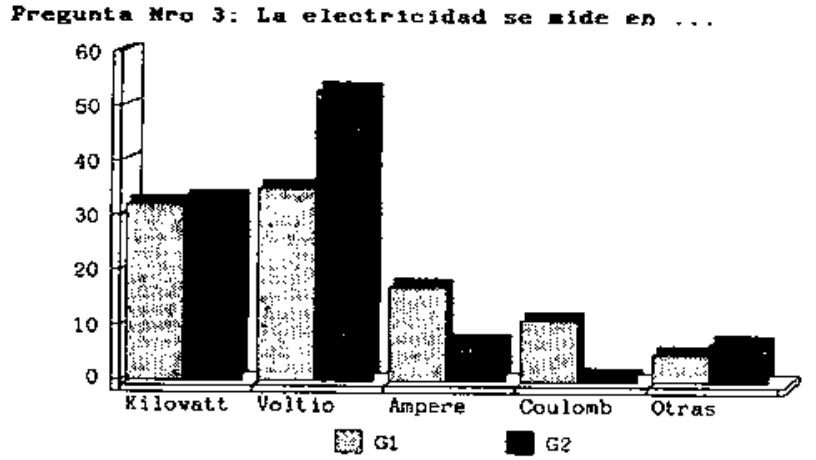

Respuesta a la pregunta $n^{\circ} 4$
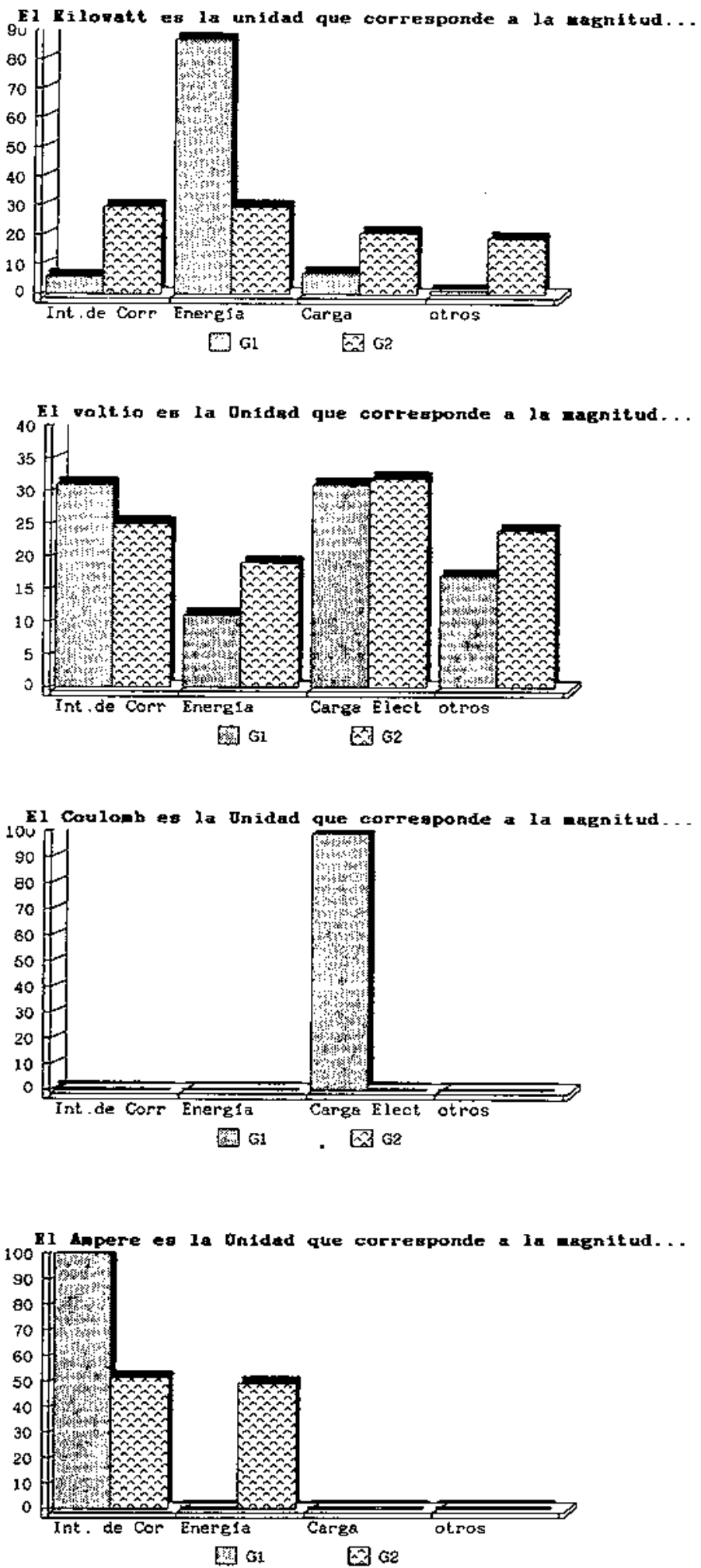
Respuesta a la pregunta $n^{\circ} 5$

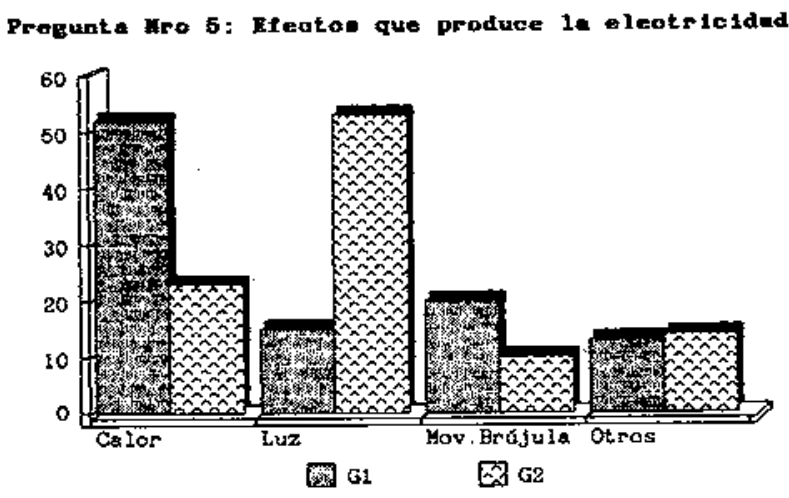

Respuestat at lat prestatita it"

Pregunta Mro 6: Inetrumento que aide la electricidad

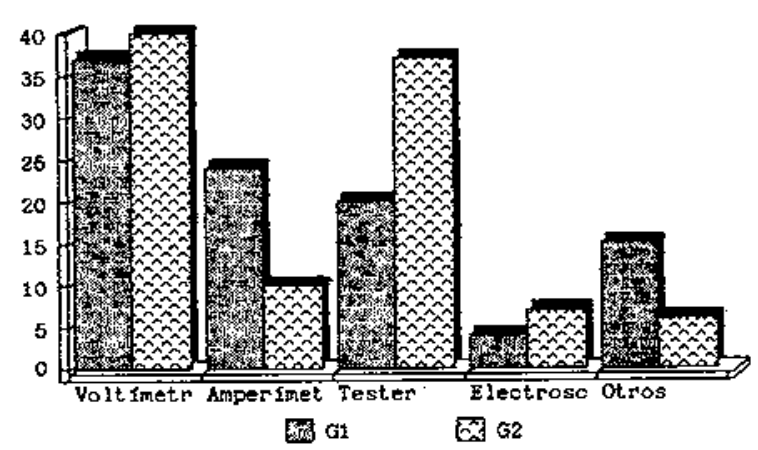

\section{REFERENCIAS BIBLIOGRÁFICAS}

AUSUBEL, D., 1973. Algunos aspectos psicológicos de la estructura del conocimiento, Simposio Phi Delta Kappa. Illinois 1963. Recopilación Elam. (El Ateneo, Bs. As.).

CLEMENT, J., 1982. Student preconceptions in introductory mechanics, Am. J. Phys. 50 (1), pp. 66-71.

CUDMANI, C., 1978. Fundamentos de Mecánica y Calor. (Libreria Universitaria: Tucumán).

CUDMANI, L. y LEWIN, A.M., 1987. Estrategias y ejes estructurales para planificar un laboratorio de física básica. Inédito. Enviado al Congreso Interamericano de Enseñanza. de la Física. Oaxtepec. México.

FEYNMAN, R., 1963. Física Vol. I. (Addison-Wesley: California).

HALBWACHS, 1985. La Física del profesor entre la Física del físico y la Física del alumno, Revista Enseñanza de la Físi$c a$, Vol. I (2).

INGARD, V. y KRAUSHAER, V., 1966. Introducción a la Mecánica: Materia y Ondas. (Reverté).

MOREIRA, M.A., 1983. Uma abordagem cognitivista ao ensino da Fisica. (Editora Universidade: Porto Alegre, Brasil).
MOREIRA, M.A. et al. Aprendizagem: Perspectivas teóricas. (Eduni-Sul UFRGS: Porto Alegre, Brasil).

PIAGET, J., 1970. Seis estudios de Psicología. (Seix Barral: Barcelona).

PIAGET, J., 1973. Psicologia de la inteligencia. (Psique, Bs. As.).

PIAGET, I., 1979. Las relaciones entre el sujeto y el objeto en el conocimiento físico-Epistemología de la Física. Vol. IV. (Paidos, Bs. As.).

POSNER, G.J. et al,, 1982. Accommodation of a Scientific Conception: Toward a Theory of Conceptual Change, Science Education, 66(2), pp. 211-227. John Wiley and Sons Inc.

SOLÍS VILA, R., 1984. Ideas intuitivas y aprendizaje de las ciencias, Enseñanza de las ciencias, Vol. 2(2), pp. 83-89.

VIENNOT, L., 1979. Spontaneous reasoning in elementary dynamics, Eur.J. Sci. Educ., pp. 205-221.

VIENNOT, L., I 985. Analysis student's reasoning: Tendencies in interpretation, Am. J. Phys., 53(5), pp. 432-486. 